Research Article

\title{
Satellite Forensics: Analysing Sparse Beacon Data to Reveal the Fate of DTUsat-2
}

\author{
René W. Fléron (D) \\ DTU Space, Technical University of Denmark, Kgs. Lyngby DK-2800, Denmark
}

Correspondence should be addressed to René W. Fléron; rwf@space.dtu.dk

Received 15 June 2018; Revised 10 December 2018; Accepted 20 January 2019; Published 5 May 2019

Academic Editor: Jeremy Straub

Copyright @ 2019 René W. Fléron. This is an open access article distributed under the Creative Commons Attribution License, which permits unrestricted use, distribution, and reproduction in any medium, provided the original work is properly cited.

\begin{abstract}
The CubeSat DTUsat-2 was designed and built by students and faculty at the Technical University of Denmark and launched to low earth orbit on June 2014. Its mission was to aid ornithologists in bird migration research. Shortly after launch and orbit injection, it became apparent that all was not nominal. To understand the problem and find the causes, a forensic investigation was initiated. The investigation used recorded Morse-encoded beacons emitted by the satellite as a starting point. This paper presents the real-life data from DTUsat-2 on orbit and the methodologies used to visualize the key element in the investigation, namely, the correlation between orbit position and the beacon counter. Based on the data presented, an explanation for the observed behaviour of DTUsat-2 is given.
\end{abstract}

\section{Introduction}

To date, more than 399 CubeSats have been launched not including the CubeSats from Planet and Spire. Depending on the success criteria applied, their failure rate is between 30 and $60 \%$ [1]. Thus, understanding the failure mechanism and not least, the method of revealing the underlying cause is relevant for the growing CubeSat community. This paper presents the investigation conducted on DTUsat-2, the recorded data, the developed method of visualizing the data, and the conclusions based on our findings.

On 19th of June 2014 at Yasny launch complex in Russia, the student satellite DTUsat-2 was launched into low Earth orbit at 19.11.11 UTC together with 32 other satellites.

During the Launch and Early Operations Phase (LEOP), it became apparent that the satellite did not behave nominally; we were thus not able to command the satellite. Figure 1 visualizes two segments of the mission timeline. The top part shows the launch from Yasny (Y) deployment sequence (D) and first pass over DTU ground station which occurred during first orbit; the dashed lines indicate ground station contact window. The lower part shows an example of the expected behaviour of the satellite after the attitude control system has locked the satellite in synch with the Earth magnetic field. The three consecutive passes are a lower eastern, a zenith, and a lower western pass. The expected beacon pattern is displayed above the three contact windows. The letter " $\mathrm{c}$ " denotes an arbitrary beacon count number between 0 and 32767. As indicated, we expected the counter to steadily increase to 32767 after which it would roll over to 0 again. Also, we expected every second beacon to originate from the on-board computer (OBC) indicated with an "o". Rather than the expected behaviour, we observed that " $c$ " had a value close to zero, i.e., between 0 and 22. The highest observed counter value, 287, occurred during the first few days of the mission. Also, the OBC beacons were often missing.

\section{Mission and Project Background}

The DTUsat-2 project was initiated in 2005 as a combined research and education project at the Technical University of Denmark. The primary mission was chosen by an external jury in a national call for payload competition. The winning proposal aimed at studying and understanding bird migration-in particular that of the cuckoo (see Data Availability section for links to further information). Cuckoos are parasites; thus, chicks are hatched and raised by another bird 

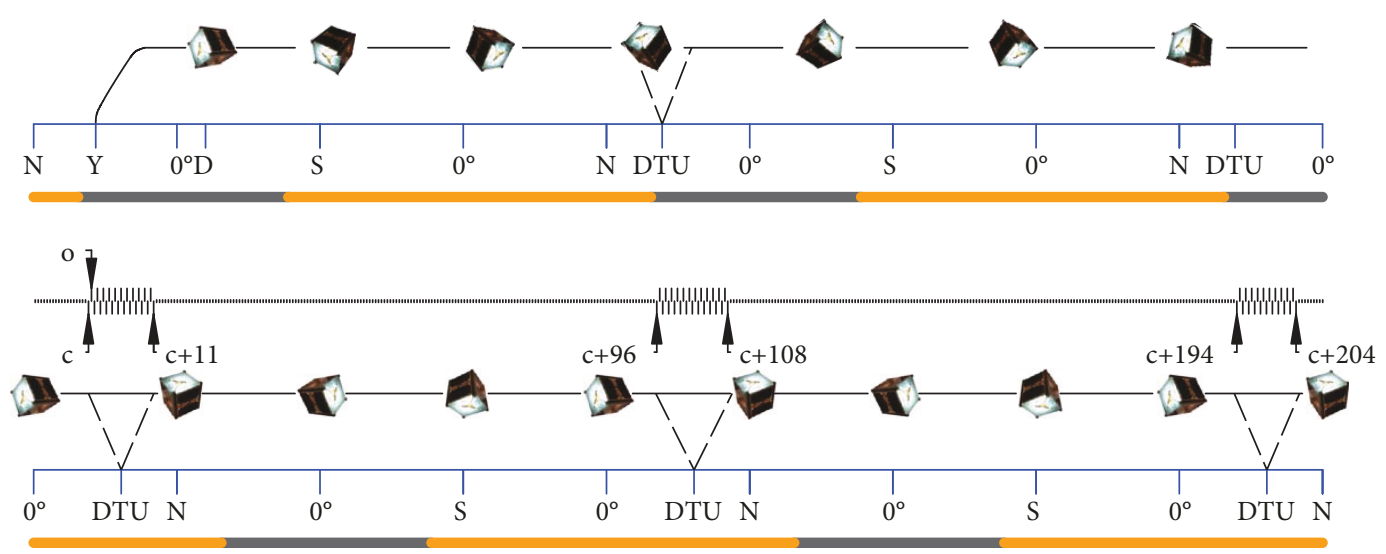

FIGURE 1: Two segments of the mission timeline for DTUsat-2. The top part shows the launch sequence from Yasny launch complex in Russia $(\mathrm{Y})$, the satellite deployment (D) and satellite contact during first orbit, which passed over DTUsat ground station (indicated with dashed lines). The lower part shows the expected behaviour of the satellite after the attitude control system has locked it to the Earth magnetic field. The letter " $c$ " indicates an arbitrary beacon count number between 0 and 32767 in the communication system beacon. During the three consecutive passes, nominal behaviour dictates that the number should steadily increase until it rolls over to zero at 32767 . The letter "o" indicates the expected on-board computer beacons, which should occur in between the communication system beacons. N, S, and $0^{\circ}$ indicates North, South, and equator, respectively. The yellow and grey bar indicates daylight and eclipse, respectively.

species. The satellite should help answer the question of how the young inexperienced cuckoo migrates $5000-6000 \mathrm{~km}$ alone at night from northern Europe to sub-Sahara Africa.

The way the bird migration question was formulated by PI Kasper Thorup essentially allowed that the satellite functioned as a simple store and forward relay of relatively small data packages $(\sim 0.8 \mathrm{kB})$ containing position data of the migrating birds. It did however require that in conjunction with the space segment (the satellite), a custom made transmitter to be strapped to the bird also had to be developed. These transmitters were named "ground segment primary payload" (GPPL).

Experience and heritage from the DTUsat-1 project launched in 2003 [2] determined the one unit (1U) CubeSat format $[3,4]$ and suggested body-mounted fixed antennas. In order to fit quarter wave antennas on the $1 \mathrm{U}$ satellite format, the communication system was designed for the $\mathrm{L}$ and $\mathrm{S}$ band, 1.2 and $2.4 \mathrm{GHz}$, respectively.

The full system comprises three major parts as follows:

(i) a number of GPPLs, one on each cuckoo to be tracked

(ii) a space segment (DTUsat-2)

(iii) a ground station for TC and TM

Figure 2 depicts the envisioned ConOps.

2.1. Satellite Subsystems. The analysis presented in this paper particularly pertains to four subsystems; these are the electrical power subsystem (EPS), the attitude control system (ACS), the on-board computer (OBC), and the communication system $(\mathrm{COM})$; a brief introduction is given below. The full satellite systems have previously been described in details elsewhere [5-7].

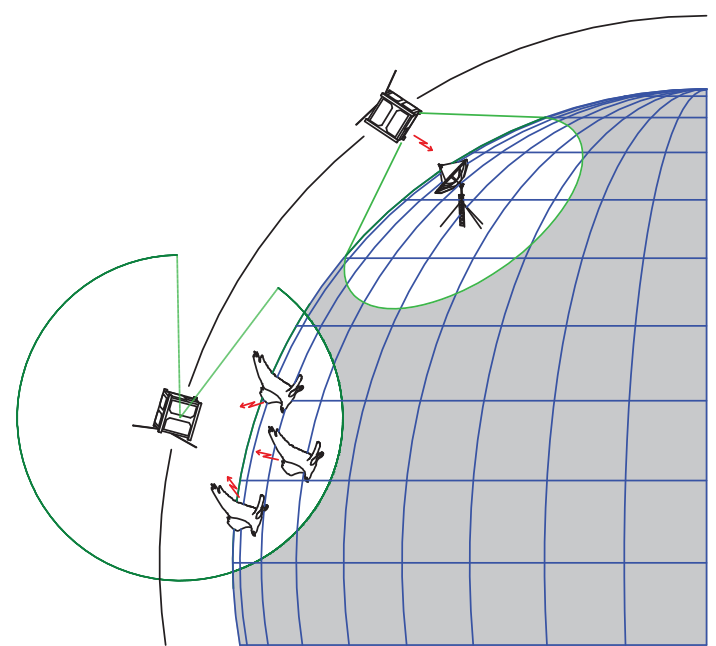

Figure 2: The ConOps of the DTUsat-2 system. The GPPLs are carried by migrating cuckoos, the PPL is on-board the space segment (DTUsat-2), and the GS handles the mission operation and data reception. Antenna coverage of both the payload and the COM system are indicated.

2.2. The Electrical Power System. The EPS consists of four sub-subsystems: the solar cell arrays (SA), the powerregulating unit (PRU), the power-supplying unit (PSU), and the power storage unit (batteries) as depicted in Figure 3. The solar array generates electrical power which is conditioned by the PRU before it either supplies the satellite through the PSU or charges the batteries. In order to save mass and volume, the lithium-ion technology was chosen for the batteries. While Li-ion has a high energy to mass fraction, the technology also imposes constraints on the satellite design. Li-ion batteries should preferably be kept above $0^{\circ} \mathrm{C}$ during operations, though some missions have successfully 


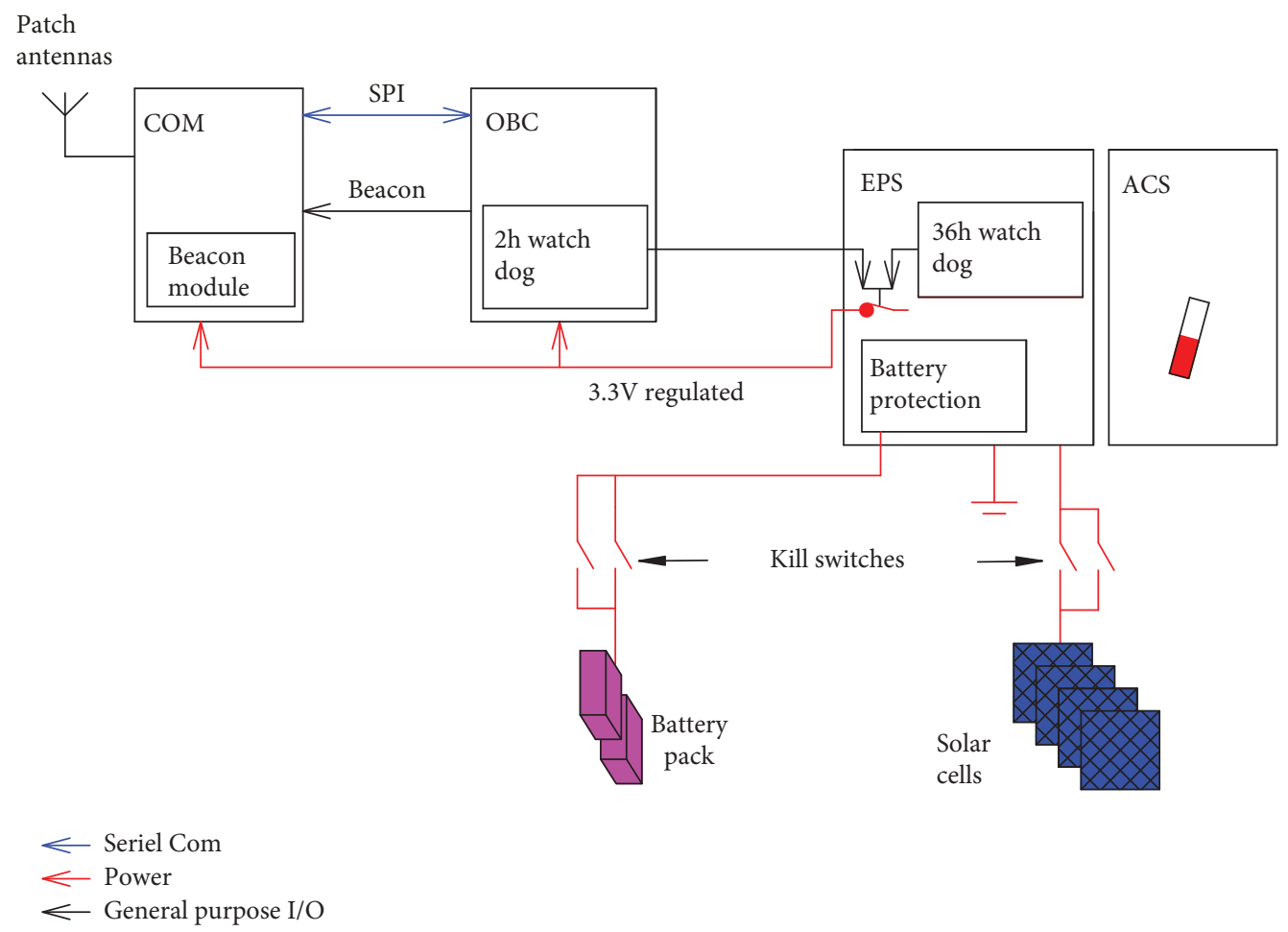

FIGURE 3: Schematics of the EPS system. Once the kill switches are deactivated at deployment, the EPS, COM, and OBC systems are powered. Two watch dog timers have been implemented, one in the OBC SW with a $2 \mathrm{~h}$ period and one in the EPS with a $36 \mathrm{~h}$ period. Beacons are generated by the $\mathrm{OBC}$ and the COM systems separately; the systems take turns in transmitting their beacons.

operated batteries below, e.g., SwissCube [8]. Further, the lifespan of the batteries depends on the depth of discharge [9]. To maximize the lifespan, a small circuit protects the batteries from both overcharging and deep discharge. In the design requirements, it was laid down that the satellite should remain power positive, i.e., have surplus power at all instances during operations. Thus, the satellite should be operational in the event that the batteries fail provided that it is illuminated by the Sun. To keep the satellite unpowered during launch, two sets of kill switches disengage the batteries and solar cells as long as the satellite is stowed in the launcher adapter. Once the satellite is deployed, a springloaded mechanism allows the switches to engage the batteries and solar cells. Two switches are implemented in each branch for redundancy.

2.3. The Attitude Control System. The initial design specification entailed an active attitude control system based on Sun sensors [2, 10], magnetometers, magnetotorquers, and a gravity gradient boom [11]. However, as the project evolved, it became apparent that neither the physical resources of a 1 unit CubeSat nor the project resources allowed an active system. Instead, a much simpler passive attitude control system was implemented. It consists of a permanent magnet and a set of libration dampening slabs made of magnetically soft $\mu$-metal, inspired by the KySat-1 mission [12].

This simplification was based on the finding that the tracking of cuckoo birds needed neither accurate attitude determination nor control. As long as the primary payload (PPL) and TC/TM antennas had a reasonable pointing accuracy the mission could be undertaken. The opening angle of the TC/TM antenna is $+/-60^{\circ}$, and the PPL antennas have a $97 \%$ spherical coverage, as illustrated in Figure 2 [5]. The passive attitude system is depicted in Figure 4.

2.4. The COM Beacon. The COM beacons are Morse encoded. This approach was chosen because it allows radio amateurs around the world to retrieve data from the satellite. At the same time, the Morse code beacon acts as a fall back for rudimentary housekeeping data because it is generated independent of the $\mathrm{OBC}$ and may be decoded manually without the help from, e.g., modems or software algorithms. Additionally, this channel for data transfer is less susceptible to noise. The data may be retrieved even if the signal-tonoise ratio (SNR) is negative. The standard hearing in noise test (HINT) reports the lower limit of speech recognition for a person with normal hearing to be $-3 \mathrm{~dB}$ [13]. However, our practical experience and SNR test have shown that the single tone Morse beacon may be decoded even at SNR levels of $-9 \mathrm{~dB}$ or less. In contrast, the noncoherent FSKmodulated OBC beacon requires a SNR of $+10 \mathrm{~dB}$ at a comparable $10^{-2}$ bit error rate. Figure 5 shows an example of a noisy beacon from a pass recorded on 20th of May 2015 with pass start at 6:59 UTC. Despite the noise, the beacon was successfully decoded.

2.5. DTUsat-2 Power Cycling/Nominal Satellite Behaviour (Operations). If either one of the four kill switches deactivates, the EPS will power up OBC and COM, which then commences emitting beacons at fixed intervals. During 


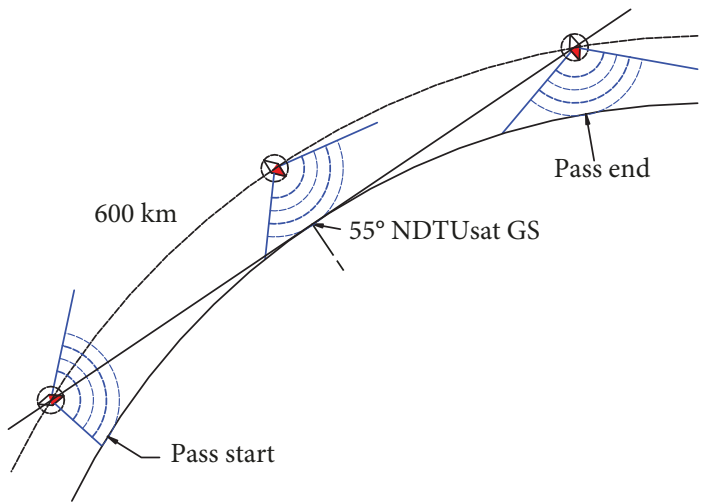

(a)

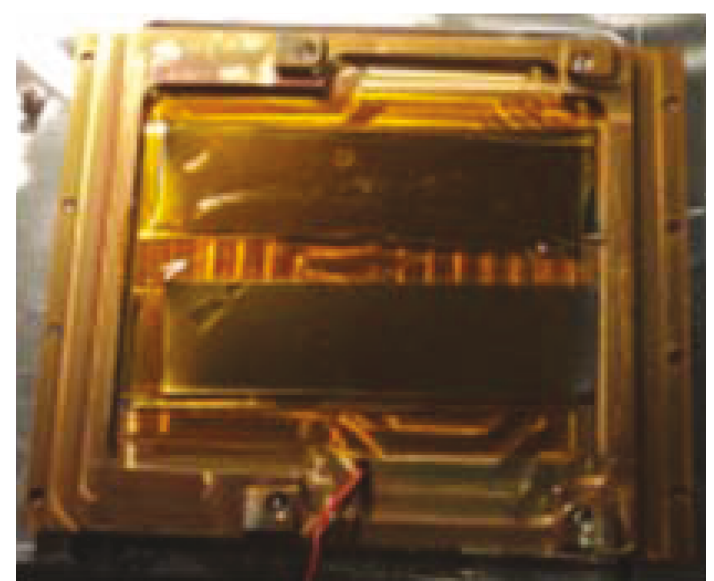

(b)

FIgURE 4: Illustration of how the descoped passive ACS system orients the satellite. The blue lines indicate the $3 \mathrm{~dB}$ opening angle of the COM system antenna. (b) Image shows the $\mu$-metal libration dampers mounted on one of the satellite panels.

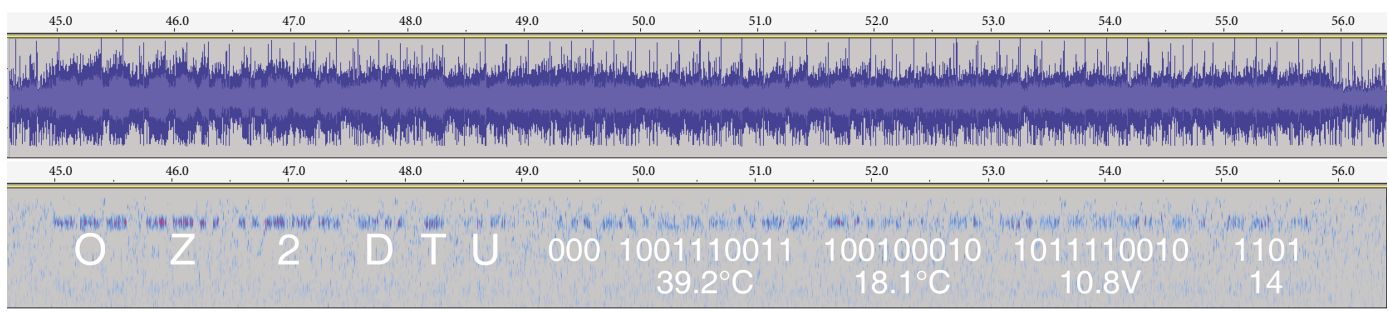

Figure 5: An example of a COM beacon recorded on $20^{\text {th }}$ May 2015. The top image shows a noisy signal. Lower image show the pitch in the signal and the decoded signal. Despite the noise the human ear may pick out the signal showing the strength of the Morse coding.

nominal operation, the beacons alternate between an $\mathrm{OBC}$ and a COM generated beacon. A beacon is transmitted every 30 seconds. The OBC beacon is FSK modulated lasting less than one second, and the COM beacon is OOK modulated lasting between 10 and 13 seconds. Both beacons contain housekeeping data. The COM specifically relays battery temperature, COM temperature, battery voltage, and beacon count number. The beacon count is incremented by one for each successfully emitted beacon. Once the counter reaches 32767 , it rolls over and starts at zero again. Thus, if all is nominal, the counter should roll over every 22.5 days. However, if for some reason the satellite is power cycled then the counter is reset to zero.

After a successful deployment, there are only three ways to power cycle the satellite: either through one of the two watch dogs or by occulting the solar cells on the $\mathrm{X}$ and $\mathrm{Y}$ panels while the battery voltage is low. Since the low beacon count number clearly indicates that power cycling does occur, the three ways of power cycling are examined.

To mitigate possible software latch-up caused by for instance single event upset or direct software code errors, two watch dogs have been implemented. Both watch dogs are capable of power cycling the satellite in order to provoke a reboot. The EPS watch dog has a $36 \mathrm{~h}$ period and is thus not considered to be causing the cycling as this happens much more often (with periods of seconds or minutes). The second watch dog is implemented in the OBC SW and thus requires the $\mathrm{OBC}$ to be running nominally. We have experienced the power cycling both with and without the $\mathrm{OBC}$ in nominal mode. Hence, the observed power cycling must be a result of solar cell occulting with low batteries.

DTUsat-2 has solar cells on four of its six sides, as depicted in Figure 6. The $+\mathrm{Z}$ panel is used for the PPL antenna; the $-Z$ panel is fitted with the TC/TM COM antennas and should be oriented towards the DTUsat ground station during passage. The $+/-\mathrm{X}$ and $\mathrm{Y}$ panels carry solar cells.

2.6. Power Usage. Prior to launch, the power consumption of the satellite was measured for the various operation modes. In idle mode, the major power usage occurs when the RF power amplifier in the COM system is active. As the OBC beacons are much shorter than the ones generated by COM, transmitting an $\mathrm{OBC}$ beacon requires much less energy. When the $\mathrm{OBC}$ operates nominally, the beacons alternate between $\mathrm{OBC}$ beacon and COM beacon. However, if the OBC have not booted correctly, it will not use its beacon slot and the COM system automatically takes over this slot. This means that overall power usage in idle mode depends on whether OBC is operating nominally or not. Power usage is displayed in Table 1.

2.7. Troubleshooting Method. The task at hand essentially consisted of three major parts as follows: 


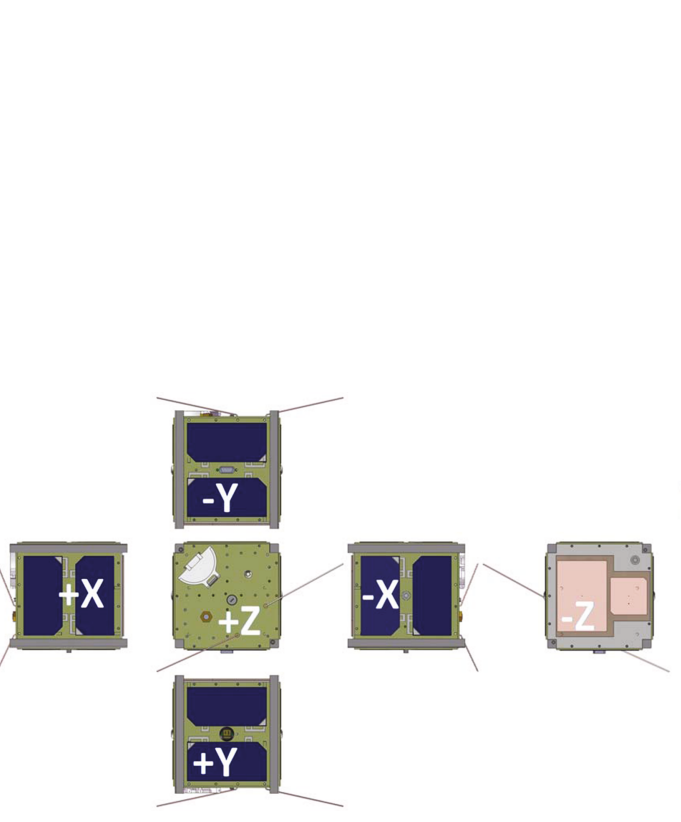

(a)

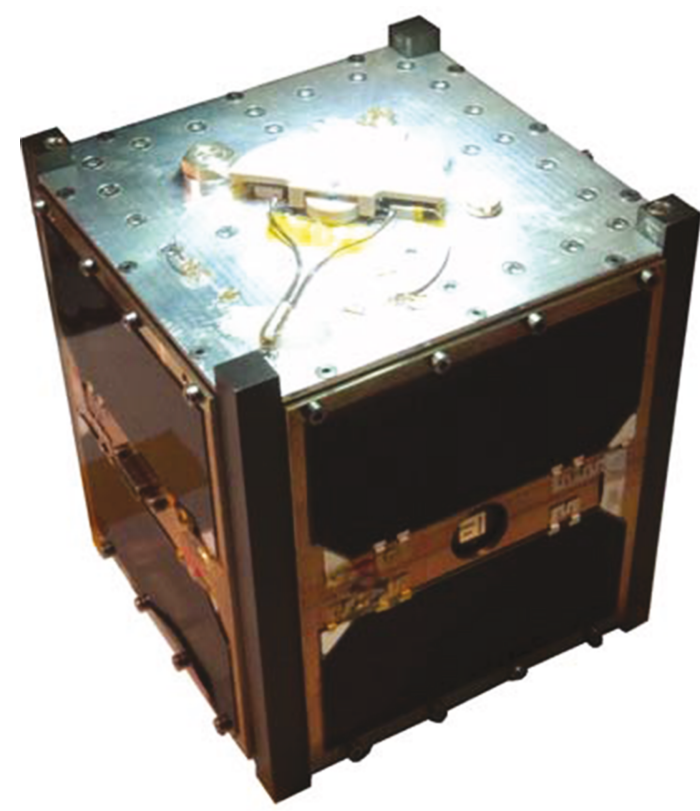

(b)

FIgURE 6: The configuration of DTUsat-2 panels. (a) The image shows an unfolded CAD rendering of the six panels. The X and Y panels are used for solar cells, the $\mathrm{Z}$ panels are used for the two antenna systems. (b) The image shows an image of DTUsat-2 taken after integration was completed.

TABLE 1: The overall DTUsat-2 power consumption in various modes of operations. When the satellite payload is inactive, the modes will alternate between idle and beacon transmission.

\begin{tabular}{lc}
\hline Operations mode & $\mathrm{mW}$ \\
\hline DTUsat-2 idle mode $\left(P_{\text {idle }}\right)$ & 420 \\
Beacon transmission $\left(P_{\text {beacon }}\right)$ & 1180 \\
Primary payload idle mode & 590 \\
Primary payload active $\left(P_{\text {payload }}\right)$ & 1930 \\
\hline
\end{tabular}

(1) Record, label, and store DTUsat-2 passes. In the USA, radio amateur Michael Williams (K9QHO) kindly offered his help in recording passes over the USA. The DTUsat ground station located at the university was used to record passes over Europe. The file label contains the start time of the pass (AOS)

(2) Listen through the recordings, decode Morse beacons, and note the time of their appearance in the recordings

(3) Plot the correlated data satellite position and beacon count number

Figure 7 shows the method applied.

Figure 8 (upper part) below shows a recording of a full pass. Recording start equals satellite pass start $+/-1$ second. The time of beacon appearance in a recording is noted along with the beacon data. The appearance time is used to determine how far into a pass a given beacon was transmitted. The final step of correlating satellite trajectory with satellite pass start for the beacon count number map is described in detail below.

To date, some 650 beacons recorded from launch till June 2015 have been decoded (as illustrated in Figure 8 lower part). Not all beacons are complete as resetting occurs randomly-also in the middle of a beacon. Since the beacon count number is the last transmitted information, only a fraction of the decoded beacons is useable in this investigation. Furthermore, poor reception also yields some recorded beacon useless since it is impossible to decode them fully. In total, 59 passes contained useful information. Of these, 42 passes are over the DTUsat ground station, giving 258 data points, and 17 passes are over the ground station of radio amateur Michael Williams, K9QHO, in Indiana, USA, giving 59 data points. A select list of decoded beacon count numbers are shown in Figure 9; the full data set is shown in Appendix.

\subsection{Reestablish Orbital Position Using the Satellite Pass Start} Time. Determining a CubeSat position using ground-based measurements has been done before. For instance, detailed analysis of beacon signals has previously been used to discern multiple CubeSats in close formation [14]; likewise, both phase measurements and polarization angle measurements on the incoming RF carrier have been used for attitude determination $[15,16]$. However, these methods are not applicable in this case. The forensic analysis presented here aims at extracting information out of the beacon counter pattern, and the data consists of audio recordings. With no tools readily available, an ad hoc method has been devised.

To visualize the beacon count data in Figure 9 (and Appendix), a correlation between the satellite position and 


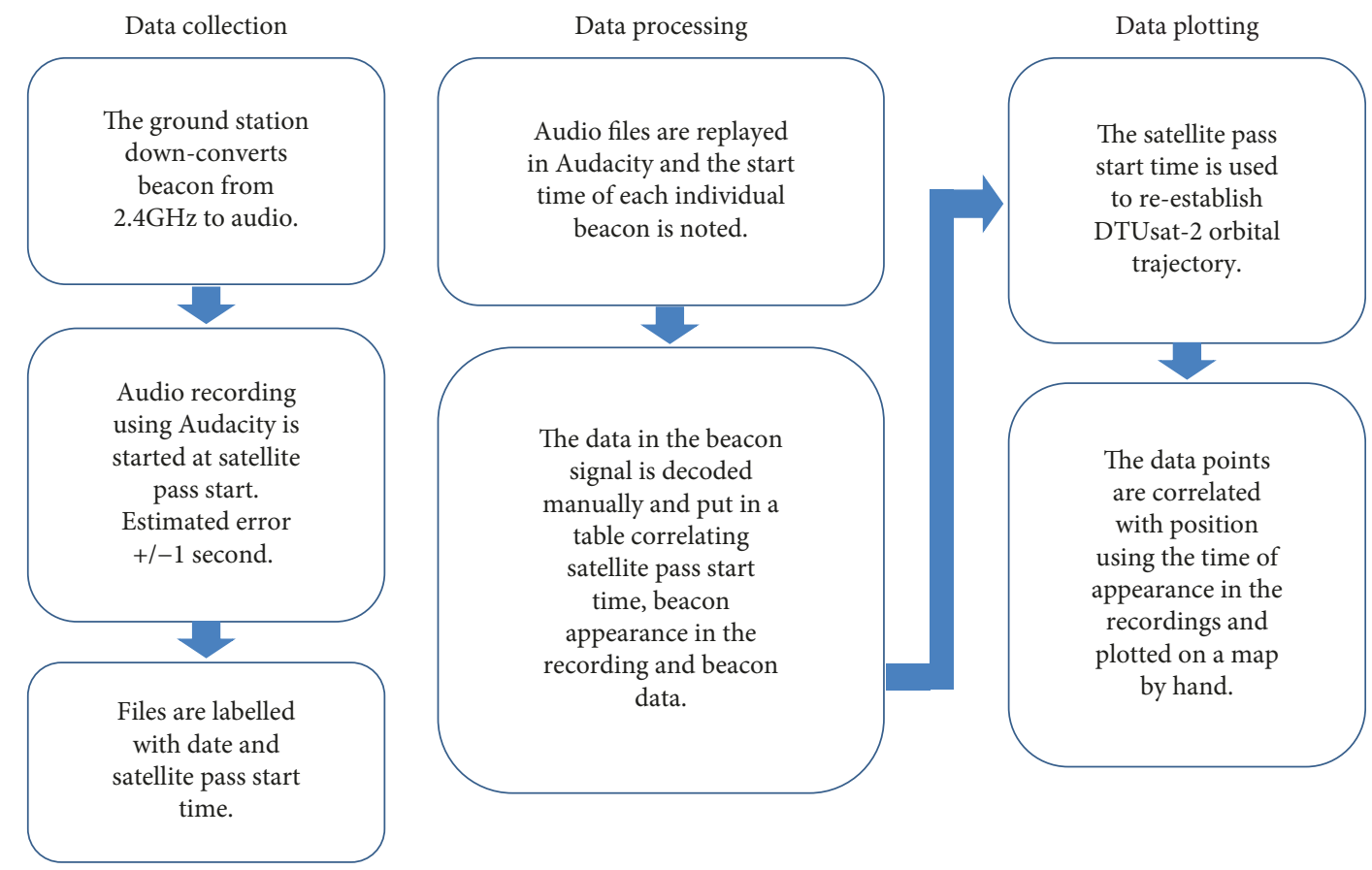

Figure 7: The figure shows a schematic representation of the data collection, processing, and plotting method. The actual data collected consists of audio files recorded during the satellite pass. The files are labelled with the pass start time (AOS). Beacon information is extracted at a later point in time by decoding the Morse beacons manually. The correlated data, position and beacon count number, is then plotted on a map.

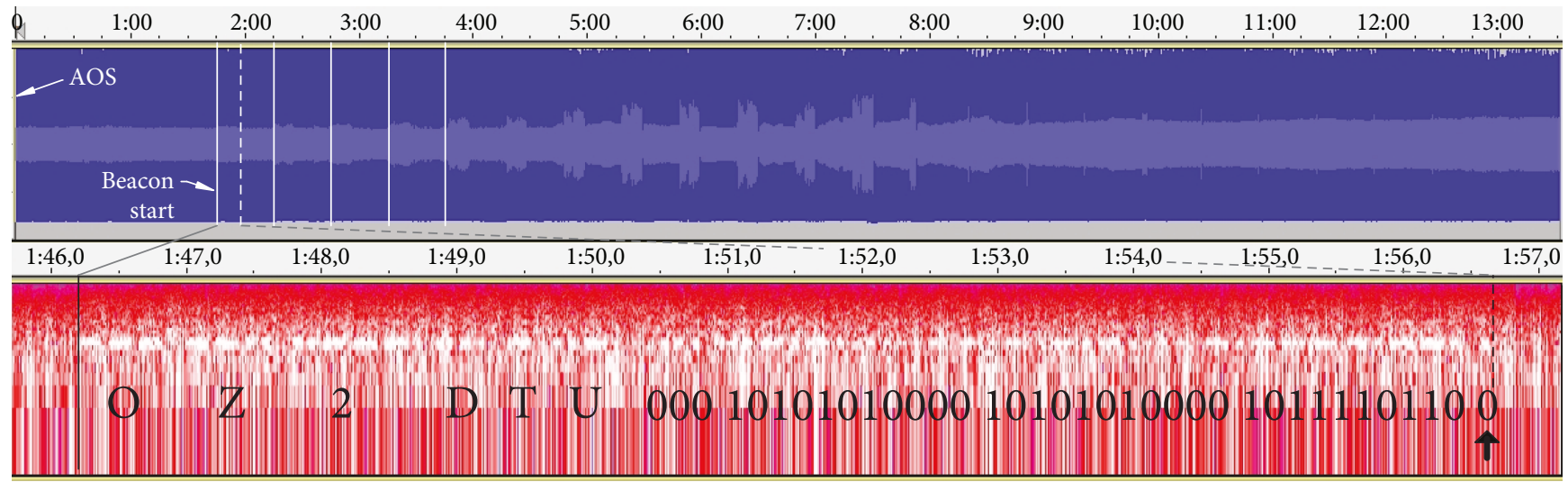

FIGURE 8: The top image shows a full pass recorded on $18^{\text {th }}$ March 2015, AOS 8:06 UTC, displayed in Audacity. The lower image is a zoom in that shows the process of decoding the first recorded beacon of the pass, which appears 1 minute and 46 seconds into the pass. The arrow at the end of the beacon indicates the beacon count number.

the beacon count number is necessary. Accurate positioning of a satellite requires a full two-line element set (TLE) and orbital visualization software. Figure 10 illustrates the full description of a satellite orbit. Although the TLEs of DTUsat-2 are known, correlating this information with the beacon count number to produce a beacon count map is cumbersome. However, since the orbit is deterministic, establishing a map with predetermined possible orbits would simplify the data mapping. We thus need to lock the orbital patterns to a map of Earth. To do this, we may utilize that the orbit is Sun synchronous and therefore deterministic. A
Sun-synchronous orbit means that the orbital plane vector is locked with respect to the Sun-Earth pointing vector. Similarly, time on Earth is defined by the Sun-Earth pointing vector, grey and yellow vectors, respectively, in Figure 10. Thus, knowing the time of satellite pass start allows for unambiguous determination of a pass trajectory. Figure 11 shows a sketch of the correlation between the DTUsat-2 orbit and the time on Earth. The purple ellipse indicates the projected field of view of a ground station, i.e., it indicates when a satellite on orbit at a given altitude becomes visible from that ground station. As a satellite passes over the ground station, 


\begin{tabular}{|c|c|c|c|c|c|c|c|c|c|c|c|c|c|c|c|c|c|c|c|c|c|c|c|c|c|c|c|}
\hline \multirow[b]{2}{*}{ Year } & \multirow[b]{2}{*}{ Month } & \multirow[b]{2}{*}{ Day } & \multicolumn{3}{|c|}{ AOS in UTC } & \multicolumn{2}{|c|}{ Beacon start } & \multicolumn{5}{|c|}{ Beacon count number } & & & & & & & & & & & & & & & \\
\hline & & & $\mathrm{h}$ & $\min$ & DK/US & $\min$ & $\mathrm{sec}$ & & & & & & & & & & & & & & & & & & & & \\
\hline 2015 & 3 & 21 & 7 & 12 & DK & 1 & 23 & 1 & & 1 & & & 3 & 4 & 5 & 6 & 7 & 8 & 9 & 10 & 11 & 12 & 13 & & & & \\
\hline 2015 & 3 & 21 & 8 & 48 & DK & 0 & 50 & 11 & 12 & 13 & & & & 1 & & 2 & & & 3 & & & & 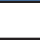 & & & & \\
\hline 2015 & 4 & 14 & 8 & 57 & DK & 2 & 42 & 1 & & 1 & & & 1 & & 1 & & & 4 & 5 & 6 & 7 & 8 & 9 & 10 & 11 & & \\
\hline 2015 & 4 & 27 & 8 & 46 & DK & 0 & 17 & 7 & & & & & & 1 & 2 & 3 & 4 & 5 & 6 & 7 & 8 & 9 & 10 & 11 & 12 & 13 & 14 \\
\hline 2015 & 5 & 18 & 16 & 46 & US & 2 & 19 & 14 & 15 & 16 & & 17 & & 18 & & & & & 1 & & & & & 4 & & & \\
\hline 2015 & 5 & 19 & 7 & 25 & DK & 1 & 25 & 1 & & 1 & 2 & 3 & & & 4 & & 5 & & & & & & & & & & \\
\hline 2015 & 5 & 19 & 17 & 0 & US & 2 & 10 & 22 & & 1 & 2 & & & & & & & & & & & & & & & & \\
\hline 2015 & 5 & 20 & 17 & 14 & US & 1 & 48 & 15 & & 16 & & & & & & & & & & & & & & & & & \\
\hline 2015 & 5 & 22 & 16 & 4 & US & 2 & 31 & 12 & & & 1 & & & & & & & & & & & & & & & & \\
\hline
\end{tabular}

FIGURE 9: The table shows a small selection of the decoded beacon data to be visualized. "Beacon start" indicates how long into the recording the first beacon appears. Each column indicates a 30 second interval. The full data set is listed in Appendix.

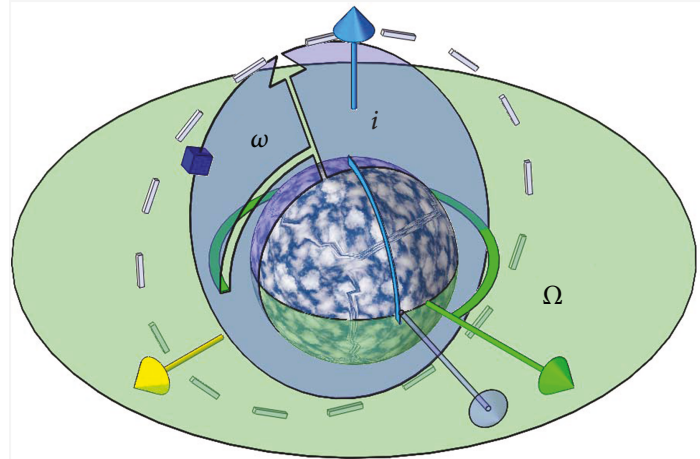

FIGURE 10: Six parameters are needed to establish a full orbital description of a satellite. However, for the Sun-synchronous orbits, a good approximation using just one parameter exists. The illustration shows the three Euler angles $(\omega, \Omega$, and $i)$ and an Earth-centered "Sun-fixed" reference coordinate system. Orbit eccentricity, mean motion, and mean anomaly are not directly indicated. The yellow Sun-Earth pointing vector fixes both time on Earth and the orbital reference coordinate system. Thus, by using satellite pass start time, it is possible to relate a pass to a given deterministic trajectory and thereby recreate the position of the satellite during a given beacon transmission, see Figure 12. The green vector is the vernal equinox vector.

the Earth rotates below; therefore, the old satellite positions indicated with open squares seem to leave the trajectory. The figure illustrates that the Earth plays the role of the hand on a clock, and the satellite orbit remains stationary like the time marks on the perimeter of a clock.

Because the orbit changes only slowly over time, the twoline element set may be used to draw out a set of template orbits on a map. These templates outline the shape of orbit trajectories, see Figure 11. The satellite pass start time may now be used to point out which trajectory the satellite is determined to follow during the pass. Thus, all we need to know to reestablish the orbital position is: what was the satellite pass start time for a given pass recording and at what time in that pass recording did the beacon appear?

This method is useful for plotting large data samples with the aim to see patterns. However, as drift occurs in the correlation between satellite pass start and azimuth over the year due to the tilted rotational axis of Earth, the method is applicable for limited time spans only. The concept is depicted in Figure 12, all times are in UTC.

2.9. Trajectory Map. The software GPredict was used to construct the basic map of trajectories and satellite pass start times. The map is created stepwise by changing the PC time manually to depict the various orbital positions and hence ground plots of the satellite fields of view as well as trajectories. By stitching the images of trajectories together, the map in Figure 12 is created. Plotting of the data points is done by hand. To aid this process, two helplines at satellite pass start +2 minutes and satellite pass start +4 minutes have been added to the map (not visible in Figure 12).

\section{Results}

Using the method described above, the correlation between the beacon count number and the satellite position may now be visualized thereby revealing any patterns between beacon count number and orbital position. Figures 13 and 14 show the results of the plotting. Each beacon count number is indicated with a small color coded square. All passes are morning passes meaning that the satellite travels from South towards North. Red squares indicate beacon number $=1$. Blue squares indicate subsequent counts, i.e., beacon count number larger than 1.

3.1. OBC Boot vs. Power. Apart from beacon number, the recorded data also reveals the status of the on-board computer. As stated previously, every second beacon should be generated by OBC (conf. Figure 2); however, our data show that this is rarely the case. The missing $\mathrm{OBC}$ beacons suggest that the on-board computer generally is not operating in its nominal mode. To better understand what causes the OBC to not boot nominally, a test set-up with the engineering model of DTUsat-2 was established. In the set-up, the power system characteristics explained in Introduction is replicated, i.e., the satellite operates without a battery running only on solar cell power. The hypothesis was that the voltage on the $3.3 \mathrm{~V}$ power bus fluctuates during power up causing the onboard computer to execute an internal factory boot sequence. From this state, the $\mathrm{OBC}$ is unable to reach nominal mode. The test showed that when the solar cell panel is slowly rotated into the sunlight, the bus voltage does indeed 

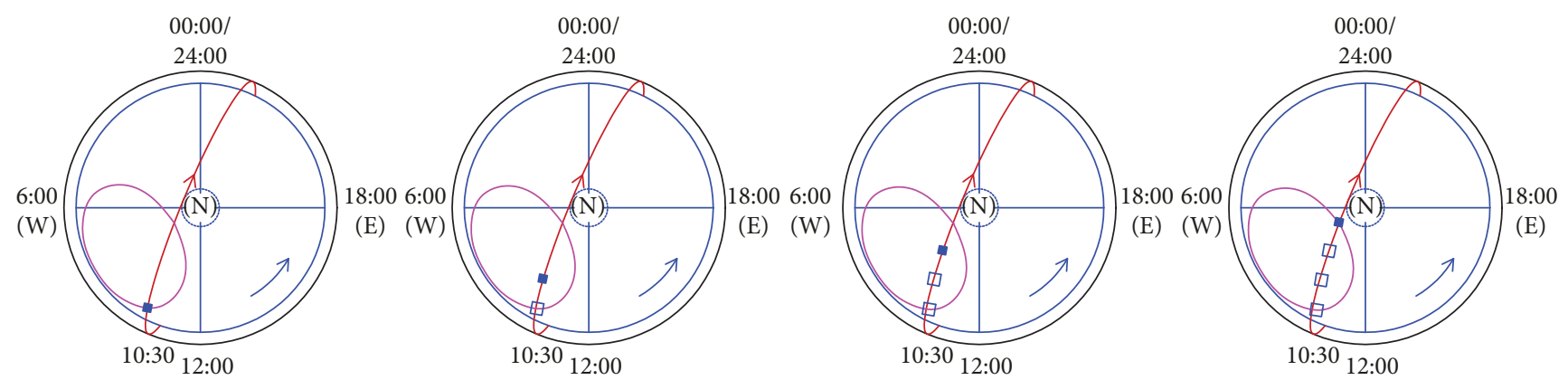

FIGURE 11: DTUsat-2 is in a Sun-synchronous orbit with a LTAN of 10:30 AM; thus, the orbital plane vector remains locked with respect to the Earth-Sun direction vector. It is the Earth-Sun direction vector that determines time on Earth, thus knowing satellite pass start time enables determination of orbit azimuth, which is used to map out the beacon count numbers.

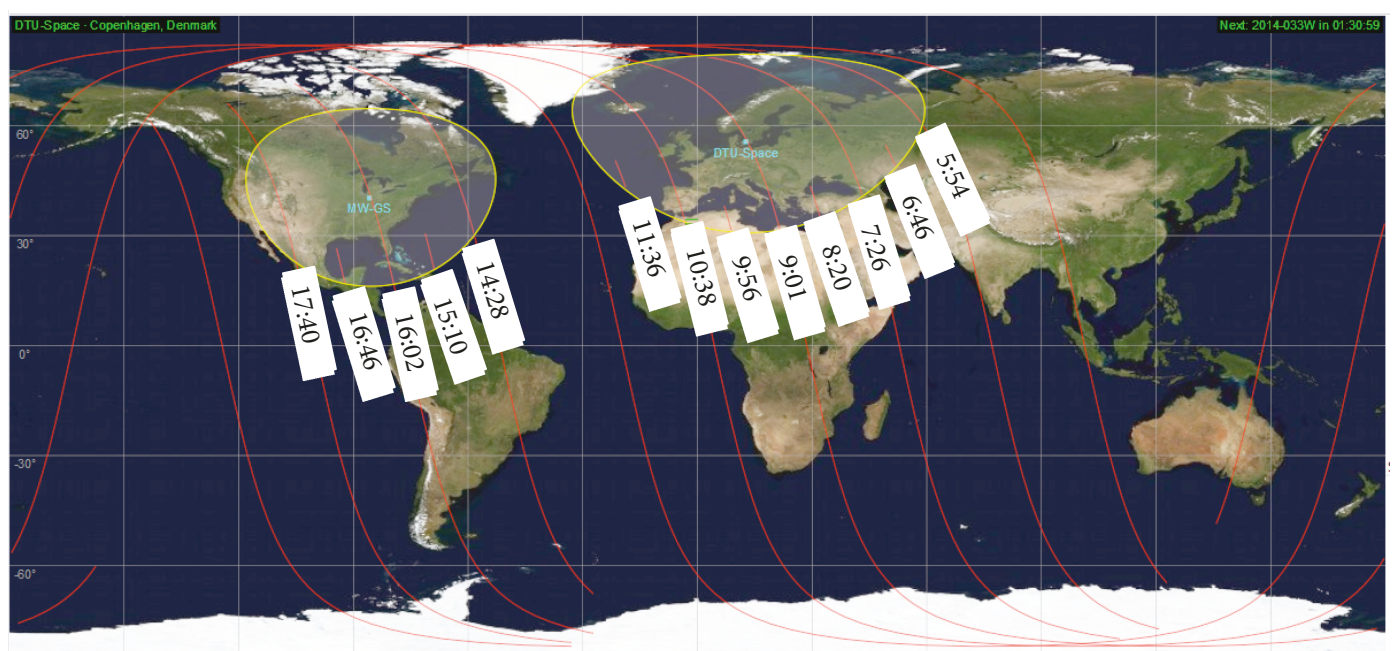

Figure 12: Map of the Earth with the two ground stations listening for DTUsat-2: one in Indiana, USA, and one in Denmark. The perimeter of each ground station is indicated with a yellow line. The red lines on the map are satellite trajectories. The time stamps at the perimeters of the field of views correspond to different satellite pass start times in UTC. Knowing the satellite pass start time allows for the determination of which trajectory the satellite will follow during the pass.

fluctuate. Figure 15(a) illustrates an eventless boot sequence with an operational battery to stabilize the $3.3 \mathrm{~V}$ power bus. Figure 15(b) shows the $3.3 \mathrm{~V}$ power bus voltage during boot using only a solar cell panel as power source. The panel is rotated by hand into the sunlight. Multiple tests with various rotational rates were conducted. High rotational rates caused less $\left(\sim 80^{\circ} / \mathrm{sec}\right)$ or no $\left(\sim 500^{\circ} / \mathrm{sec}\right)$ power fluctuation whereas lower rates caused fluctuations as seen in the graph on Figure 14(b). The DTUsat ground station was used to record the subsequent beacon pattern after a boot attempt. Not surprisingly, the battery-assisted boot sequence results in the desired pattern alternating between COM and OBC beacons. Conversely, the batteryless test results in a beacon pattern with only COM beacons, which supports the faulty boot sequence hypothesis.

\section{Discussion}

From Table 1 in Power Usage, it is seen that beacon transmission almost triples power requirements and is only supported when the solar cell panels are sufficiently illuminated. Attitude variations and satellite panel topology dictates a varying power supply from the EPS. The main variation in power generation naturally occurs with changes in the magnetic inclination during orbit. However, our investigation has shown satellite performance variations between westerly and easterly passages which are not explained by the inclination.

4.1. Isogonic Map. Data on the Earth magnetic field is available online from the National Oceanic and Atmospheric Administration (NOAA). Through the NOAA web portal, it is possible to generate a map using their historical data of isogonic lines showing the magnetic declination, see Figure 16 (see Data Availability for further information on the NOAA website). The line of zero declination is highlighted in yellow. Red lines indicate an eastern declination; conversely, blue lines indicate a western declination. Although we have listened for the satellite at both early and late passes over both Europe and the USA, it is clearly seen in Figures 13 and 14 that the recorded beacons are not evenly distributed. By comparing the plotted beacon count data in 


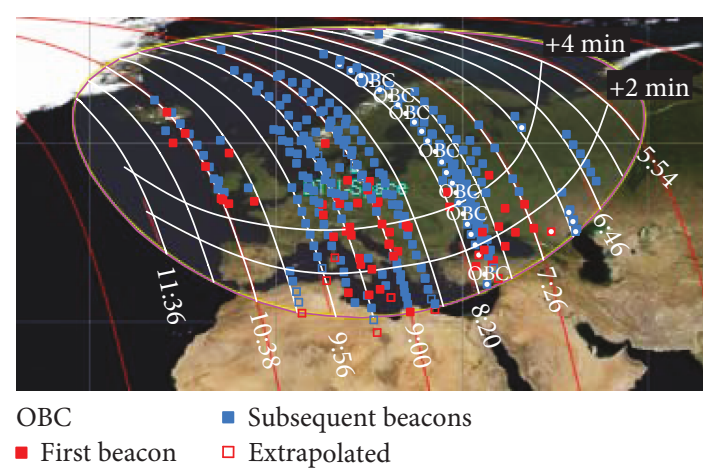

FIGURE 13: Map of the beacon counter data received by the DTUsat ground station. The white lines are guiding lines used to aid the placement of the beacon dots. Red dots indicate first beacon after a reboot. Blue squares indicate a beacon number higher than 1 . The letter group $\mathrm{OBC}$ indicates an $\mathrm{OBC}$ beacon. The white dots mark beacon from a small recording campaign a few months after the main recording campaign. All the dots are placed using the approach described in the troubleshooting methods section; however, the UTC times indicated at the perimeter are the ones that relates to the main (and largest) recording campaign.

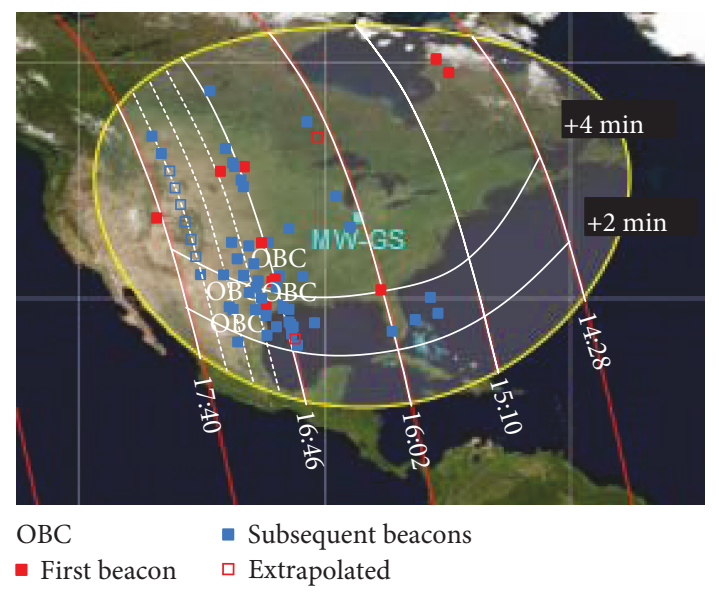

FIGURE 14: Map of the beacon counter data received by Michael Williams, K9QHO, ground station in Indiana, USA. White lines are guide lines to aid the placement of beacon dots. Red squares indicate first beacon after a reboot. Blue squares indicate a beacon number higher than 1 . The letter group OBC indicates an OBC beacon.

Figures 13 and 14 with the declination map in Figure 16, it is seen that the satellite generally sends more beacons when it flies over parts of the Earth with an eastern declination of the magnetic field. The satellite orbital plane has a LTAN of 10:30 AM; thus, the sunlight has an angle to the orbital plane of approximately $22.5^{\circ}$, disregarding that the orbit inclination is not $90^{\circ}$. Variations in the magnetic declination will cause the satellite to turn slightly. Eastern declination will turn the normal vector of the $\mathrm{X}$ and $\mathrm{Y}$ panels (with solar cells) towards alignment with the sunlight, i.e., the angle between the normal vector of a side panel with solar cells, and the solar radiation will have a lower minimum value, see the right CAD rendering on Figure 16. Conversely, a western declination will turn the satellite such that the normal vector of the solar cell carrying panels (X and $\mathrm{Y}$ ) has a higher minimum value, see left CAD rendering on Figure 16. Maximum power is generated when the angle between the panel normal vector and the sunrays is zero.

This declination variation is in good agreement with our beacon data plotted in Figures 13 and 14 that show significantly more data points in regions with eastern declination. From our analysis, we conclude that the resetting is closely connected to the behaviour of the on-board power system. We thus conclude that the batteries have failed due to the combination of a marginal power budget and wrong limit values for the battery protection circuit. Thus, the power system is only operational when the solar panels are sunlit. We have shown that there is a correlation between the inclination and the declination of the Earth magnetic field and the resetting of DTUsat-2.

These findings have further been supported by a set of experiments as follows:

(i) During the late evening passes the satellite flies from North towards South. In the summer time, the satellite will come into view of the DTUsat ground station at the university while it is still illuminated by the Sun. During the pass, it will go into eclipse. We have observed that beacons disappear as the satellite passes into eclipse

(ii) We listened for beacons during the three consecutive passes over DTUsat ground station on March 20, 2015. A solar eclipse over Europe took place on that day. During the first pass in the morning and the last pass at midday, beacons were heard. However, no beacons were heard in the second pass that went through the solar eclipse

Although the cause of the satellite behaviour in the end may seem trivial, reaching this conclusion was not. First, the resetting had to be attributed to the power system itself and not for instance the software implemented watch dog timer. Then, scrutinizing the EPS schematics revealed that erroneous resistor values in the battery protection circuit unfortunately had slipped under the radar of several reviews and tests. Finally, the missing operations of the on-board computer had to be explained since the computer and the COM system runs on the same power bus. Only by detailed mapping of the beacon data were we able to understand the behaviour and why.

\section{Conclusion}

Our investigation has led us to the conclusion that the onboard battery has been destroyed as a consequence of two errors in the satellite. Wrong value of four resistors in the power system battery protective circuitry results in too low limit values of the battery charging cycle. The passive attitude system optimized for antenna directivity results in low power generation during passage over northern Africa. In combination, this results in deep discharge of the battery with no 


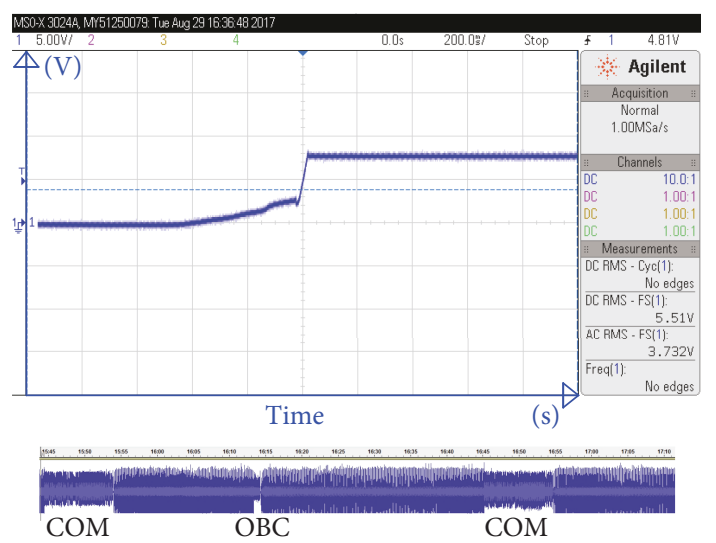

(a)

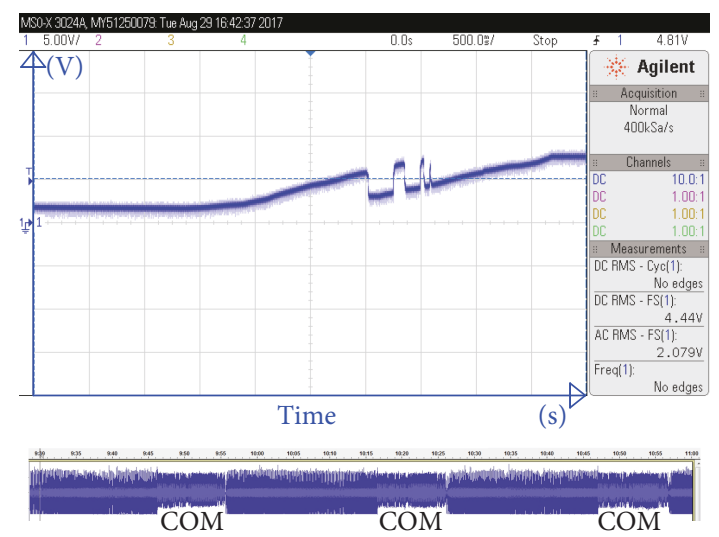

(b)

FIGURE 15: The left graph (a) shows an uneventful power-up sequence with an operational battery. Time division is $200 \mathrm{mSec}$ and voltage division is $2.5 \mathrm{~V}$. The right graph (b) shows a power-up sequence with only a solar cell panel as power source. The panel is rotated into the sunlight with a rate of approximately $36^{\circ} / \mathrm{sec}$. Time division is $500 \mathrm{mSec}$, and voltage division is $2.5 \mathrm{~V}$. Inset below both graphs are the subsequent beacon patterns recorded with the DTUsat ground station.

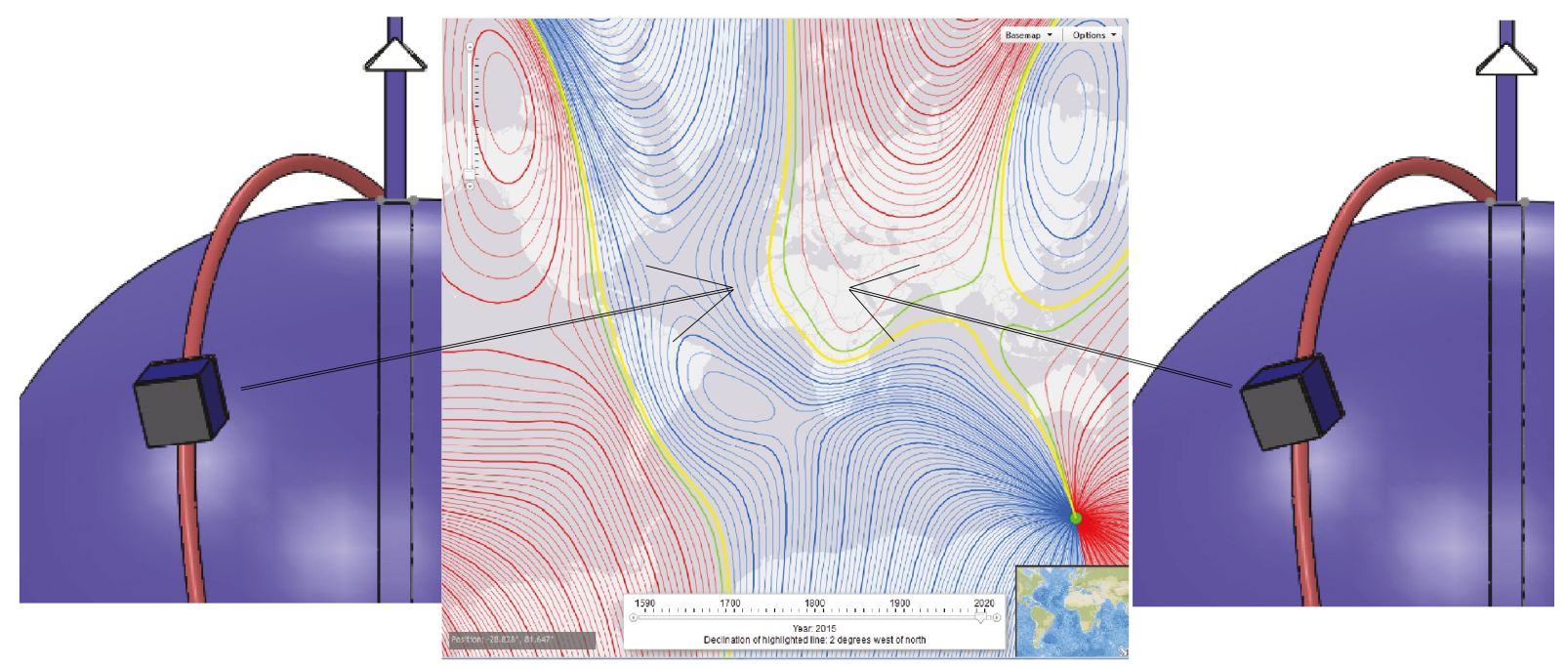

Figure 16: A map of magnetic field declination produced from historical data available at the National Centers for Environmental Information under NOAA. The yellow isogonic line indicates a shift in magnetic declination. Blue lines indicate western declination, and red lines indicate eastern declination. Whenever DTUsat-2 is west of the yellow line over Europe, we have observed lesser performance. The left CAD rendering shows the attitude effect of western declination. When DTUsat-2 is east of the yellow line, we see better performance. The right CAD rendering shows the attitude effect of eastern declination. Likewise, Michael Williams, Indiana, USA, has reported lesser performance during DTUsat-2 passes east of the yellow line over the USA.

possibility of recovery, and ultimately, the battery has suffered permanent damage. Once the battery has been discharged, the $3.3 \mathrm{~V}$ power bus voltage is not stabilized. This means that when resetting occurs either due to eclipse or magnetically induced attitude changes, the $\mathrm{OBC}$ booting sequence may fail due to voltage fluctuations of the $3.3 \mathrm{~V}$ power bus. A failed OBC boot leads to extra power expenditure as the $\mathrm{OBC}$ beacon vacancies are filled with the longer COM beacon making it even harder for the battery to recover.

The attitude system based on a permanent magnet was oriented for optimization of the RF link during a ground station pass and not power generation. With no power storage facility the operation of DTUsat-2 is governed by variations in inclination and declination of Earth's magnetic field.

5.1. Outlook. The DTUsat-2 project was initiated as a joint research and educational project. The educational objective has been met as well as selecting a genuine research project suited for the small one unit CubeSat platform. However, the operational phase has shown that system integration and testing has to be given more emphasis. To allow time for this, the satellite bus interface is now designed and 


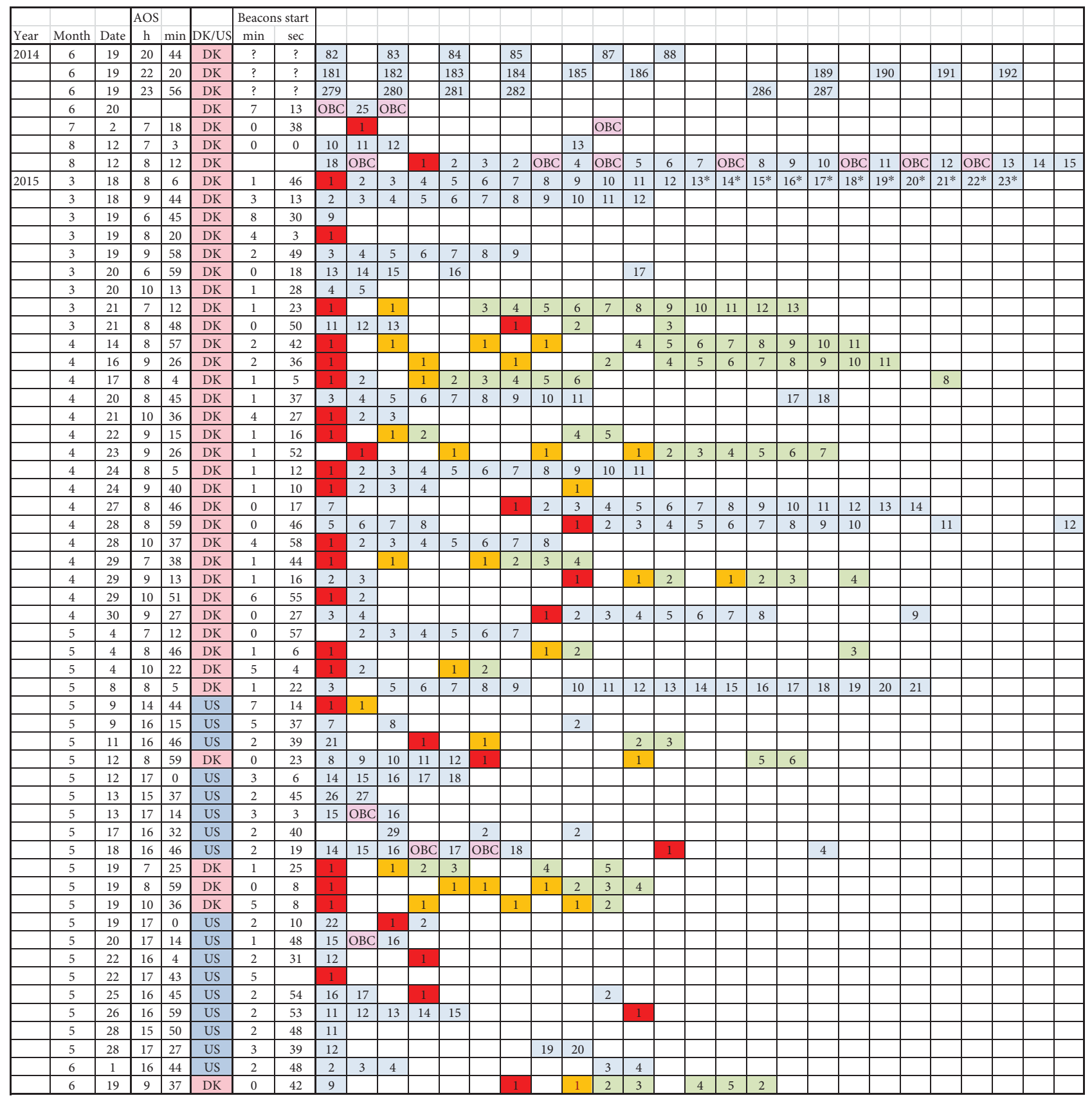

FIGURE 17

manufactured by faculty and university prior to any student subsystem project. This allows the students to test their system directly up against the final flight configuration from the very onset of their projects. The students operate and test their systems through the actual flight version of the bus hardware. This approach increases the change of revealing any unwanted side effects of subsystem interaction.

\section{Appendix}

Figure 17 presents the cleaned up beacon data set that has been used for the presented forensic analysis of DTUsat-2.

\section{Data Availability}

The beacon data used for the forensic analysis in this paper and which is presented in Appendix are also available from the corresponding author upon request. The cuckoo migration experiment as proposed to the DTUsat-2 project is described in some detail on this web address: https:// directory.eoportal.org/web/eoportal/satellite-missions/d/dtu sat-2 and the actual proposal: K. Thorup, "Solving the mystery of bird migration: Tracking small birds from space," 2005 is available at this web address: https://www.dtusat. dtu.dk/fileadmin/docs/payload/Bird_proposal.pdf. For our understanding of the human ear signal-to-noise-ratio 
threshold of Morse beacon decoding a set of audio test files has been produced. These are available at https://www .dtusat.dtu.dk at the bottom of the DTUsat-2 Launch Event page. The map of isogonic lines has been created using the National Centers for Environmental Information Historical magnetic declination website: https:/maps.ngdc.noaa.gov/ viewers/historical_declination/ (accessed June 2018).

\section{Conflicts of Interest}

The author declares that there is no conflict of interest regarding the publication of this paper.

\section{Acknowledgments}

The DTUsat-2 team would like to express their gratitude and thank radio amateur Michael Williams, K9QHO, Indiana, USA, for his generous contribution to the investigation of the DTUsat-2 behaviour. The work presented here has been funded internally by the Technical University of Denmark.

\section{References}

[1] M. Swartwout, "Secondary spacecraft in 2016: why some succeed (and too many do not)," in 2016 IEEE Aerospace Conference, pp. 1-3, Big Sky, MT, USA, 2016.

[2] J. H. Hales and M. Pedersen, "Two axis MOEMS sun sensor for pico satellites," in Proceedings of the 16th Annual AIAA/USU Conference on Small Satellites, Logan, UT, USA, 2002.

[3] I. Nason, J. Puig-Suari, and R. Twiggs, "Development of a family of picosatellite deployers based on the CubeSat standard," in Proceedings, IEEE Aerospace Conference, pp. 1-457-1-464, Big Sky, MT, USA, USA, 2002.

[4] S. Lee, A. Hutputanasin, A. Toorian et al., “"CubeSat Design Specification (CDS) Rev. 13," The CubeSat Program, Cal Poly SLO," 2014, https://static1.squarespace.com/static/5418c831e4 b0fa4ecaclbacd/t/56e9b62337013b6c063a655a/14581570954 54/cds_rev13_final2.pdf.

[5] J. B. Bjarnø and R. W. Fléron, "DTUsat-2 - the next generation animal migration research platform," in Proceedings of the $4 S$ Symposium Small Satellites Systems and Services, Rhodes, Greece, 2008.

[6] R. W. Fléron, "Mechanics of DTUsat-2," in Proceedings of the 4 S Symposium Small Satellites Systems and Services, Portoroz, Slovenia, 2012.

[7] R. W. Fléron, "A satellite based low power low volume receiver system for tracking of migrating birds," in Proceedings of the 66th International Astronautical Congress 2015, pp. 44304437, Jerusalem, Israel, 2015, IAC-15-B4.4.2.

[8] S. Rossi and A. Ivanov, "Thermal model for cubesat: a simple and easy model from the Swisscube's thermal flight data," in Proceedings of the International Astronautical Congress, pp. 9919-9928, Beijing, China, 2013.

[9] L. Lam and P. Bauer, "Practical capacity fading model for Li-ion battery cells in electric vehicles," IEEE Transactions on Power Electronics, vol. 28, no. 12, pp. 5910-5918, 2013.

[10] R. W. Fléron, M. Pedersen, J. H. Hales, P. R. Bidstrup, and A. Torp, "Two Axis MOEMS sun-sensor and electron emitter developed for DTUsat," in Proceedings 4th Roundtable on
Micro and Nano Technology for Space, pp. 1-8, ESA Estec, Noordwijk, The Netherlands, 2003.

[11] M. J. Sidi, Spacecraft Dynamics and Control: A Practical Engineering Approach, Cambridge University Press, 2002, Chapter 5.

[12] S. A. Rawashdeh and J. E. Lumpp Jr., "Nano-satellite passive attitude stabilization systems design by orbital environment modeling and simulation," in AIAA Infotech@Aerospace 2010, pp. 2010-3413, Atlanta, GA, USA, 2010.

[13] J. B. Nielsen and T. Dau, "The Danish hearing in noise test," International Journal of Audiology, vol. 50, no. 3, pp. 202208, 2011.

[14] M. Stolarski and G. Woźniak, "Estimation of PW-Sat satellite orbit based on Doppler effect," in Photonics Applications in Astronomy, Communications, Industry, and High-Energy Physics Experiments, vol. 8454 of Proceedings of SPIE, pp. 84540 H-1-84540H-6, Wilga, Poland, 2012.

[15] D. Roascio, L. M. Reyneri, C. Sansoé, and M. Bruno, "Small satellite attitude determination with RF carrier phase measurement," in 60th International Astronautical Congress (IAC-09), pp. 4857-4863, Daejeon, Republic of Korea, 2009.

[16] S. A. Parvez, "Attitude determination using antenna polarization angles," Journal of Guidance, Control, and Dynamics, vol. 14 , no. 2, pp. 236-240, 1991. 


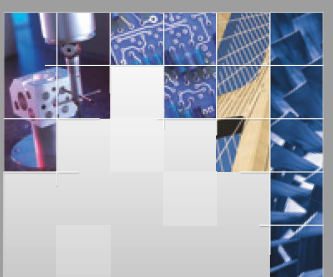

\section{Enfincering}
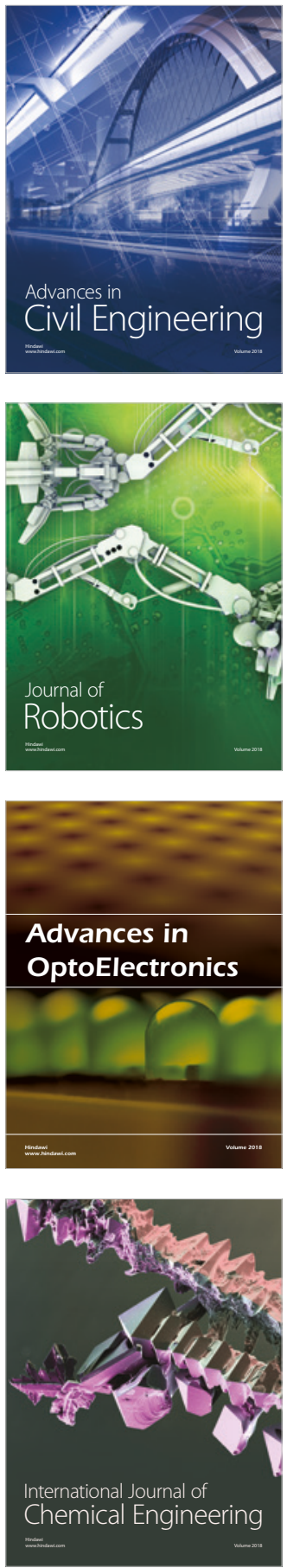

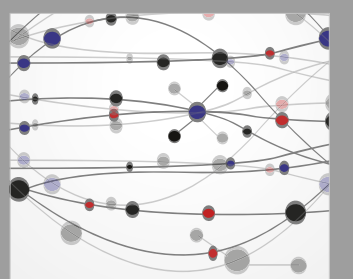

\section{Rotating \\ Machinery}

The Scientific World Journal

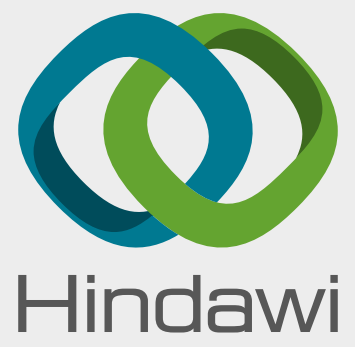

Submit your manuscripts at

www.hindawi.com
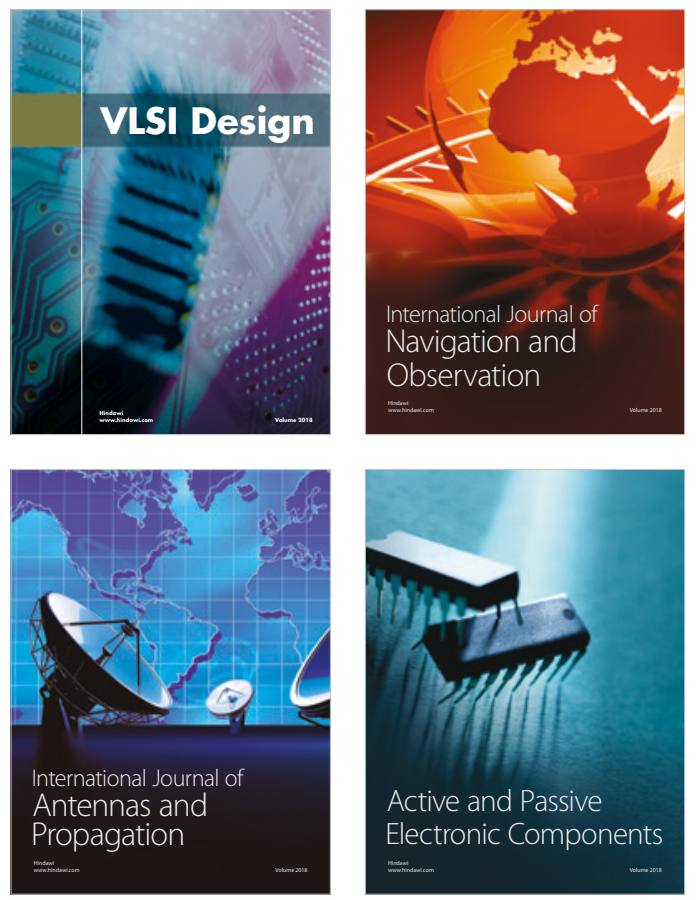
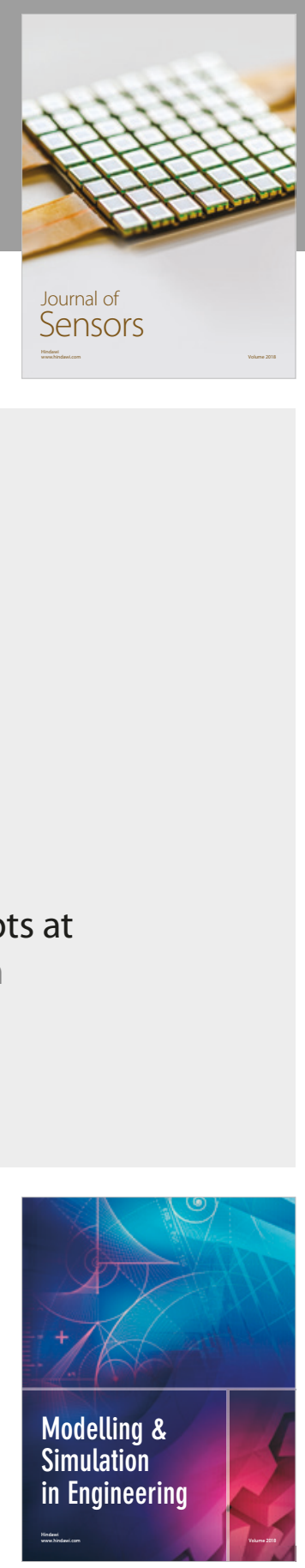

\section{Advances \\ Multimedia}
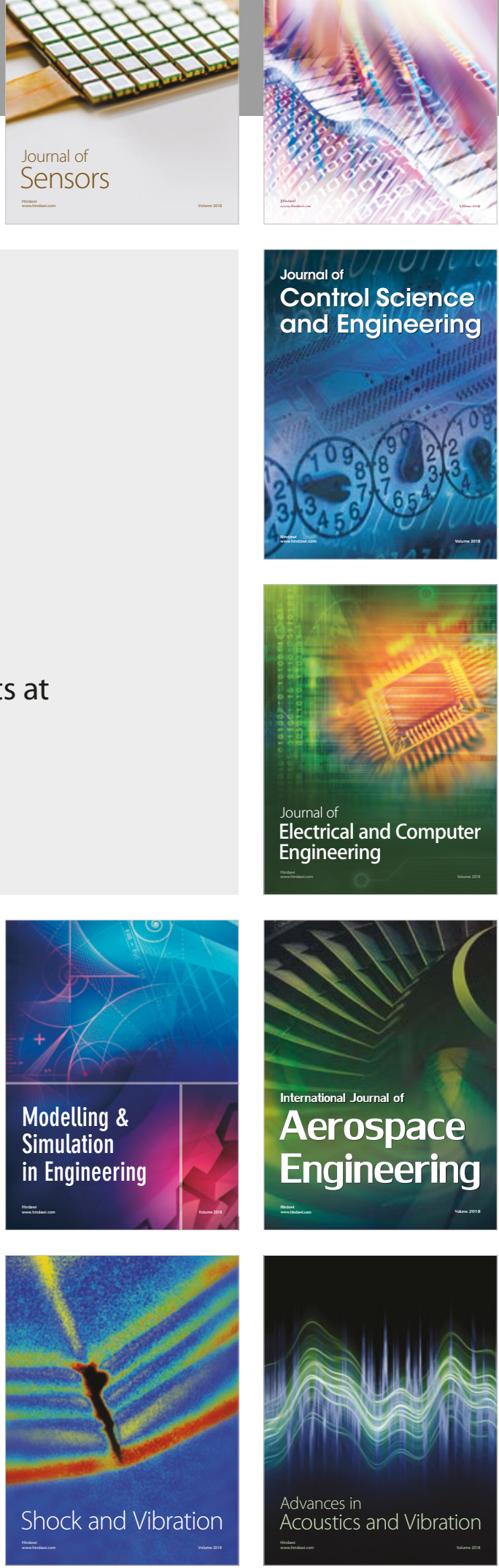